\title{
Endobronchial tumor debulking with a flexible cryoprobe for immediate treatment of malignant stenosis
}

\author{
Christian Schumann, MD, ${ }^{\mathrm{a} *}$ Martin Hetzel, MD, ${ }^{\mathrm{b} *}$ Alexander J. Babiak, MD, ${ }^{\mathrm{b}}$ Jürgen Hetzel, MD, ${ }^{\mathrm{c} *}$ \\ Tobias Merk, MD, ${ }^{\mathrm{a}}$ Thomas Wibmer, MD, ${ }^{\mathrm{a}}$ Philipp M. Lepper, MD, ${ }^{\mathrm{d}}$ and Stefan Krüger, $\mathrm{MD}^{\mathrm{a}, \mathrm{e}}$
}

\begin{abstract}
Objective: In addition to use of a laser, argon plasma coagulation, electrocautery, or coring with a rigid bronchoscope, tumor debulking with a flexible cryoprobe is used for therapeutic bronchoscopy with an immediate effect for endobronchial pathologies. We performed this analysis to determine the usefulness, efficacy, and safety of the flexible cryorecanalization in a large population under routine conditions.
\end{abstract}

Methods: We identified 225 bronchoscopic interventions that were done as cryorecanalization with a flexible cryoprobe. All patients had symptomatic airway stenosis. We determined the endoscopic success rate and safety (bleeding and perforation) of the procedure.

\begin{abstract}
Results: Successful cryorecanalization was achieved in 205 (91.1\%) of 225 patients. The flexible cryoprobe was used with all patients, in most patients in combination with flexible bronchoscopy and only in a minority $(\mathrm{n}=31,13.8 \%)$ in combination with a rigid bronchoscope. Additional interventional techniques used were endobronchial stents $(\mathrm{n}=11,4.9 \%)$ and argon plasma coagulation $(\mathrm{n}=37,16.4 \%)$. Mild bleeding (if ice-cold $\mathrm{NaCl}$ or epinephrine solution was necessary) occurred in $9(4.0 \%)$ patients, moderate bleeding (if argon plasma coagulation or a bronchus blocker was required) occurred in $18(8.0 \%)$ patients, and severe bleeding (events with hemodynamic instability) never occurred.
\end{abstract}

Conclusions: Cryorecanalization with the flexible cryoprobe for treatment of symptomatic endobronchial tumor stenosis is a safe technique with a high success rate and immediate treatment effect. (J Thorac Cardiovasc Surg 2010;139:997-1000)

Hemoptysis, cough, and dyspnea are the most frequent symptoms of patients with exophytic tumor manifestations in lung cancer. ${ }^{1-3}$ The high mortality of lung cancer is at least partially caused by locally advanced tumor growth resulting in atelectasis and pneumonia. Tumor obstruction of the central or peripheral airways can result in postobstructive pneumonia, which is difficult to treat. In such a case, a safe and effective bronchoscopic treatment modality is required to resolve symptoms and complications caused by airway obstruction. In these cases many interventional pneumologists use rigid bronchoscopy in combination with mechanical debulking with forceps, a neodymium-doped yttrium aluminum garnet (Nd-YAG) laser, electrocautery, argon plasma coagulation (APC), or brachytherapy to treat endoluminal

\footnotetext{
From the Center of Internal Medicine, Clinic of Internal Medicine II, ${ }^{\mathrm{a}}$ University of Ulm, Ulm, Germany; the Clinic of Pneumology and Internal Medicine, ${ }^{b}$ Red Cross Hospital, Stuttgart, Germany; the Department of Internal Medicine II, ${ }^{\mathrm{c}}$ University of Tübingen, Tübingen, Germany; the Department of Pneumology, ${ }^{\mathrm{d}}$ University Hospital of Bern (Inselspital) and University of Bern, Bern, Switzerland; and Medical Clinic I, ${ }^{\mathrm{e}}$ University Clinic Aachen, Aachen, Germany.

Disclosures: M.H., A.J.B., and J.H. report lecture fees from ERBE, the manufacturer of the cryoprobe.

* C.S., M.H., and J.H contributed equally to this article.

Received for publication March 16, 2009; revisions received May 9, 2009; accepted for publication June 20, 2009; available ahead of print Aug 28, 2009.

Address for reprints: Christian Schumann, MD, University of Ulm, Clinic of Internal

Medicine II, Albert-Einstein-Allee 23, 89081 Ulm, Germany (E-mail: christian.

schumann@uniklinik-ulm.de).

$0022-5223 / \$ 36.00$

Copyright (c) 2010 by The American Association for Thoracic Surgery

doi:10.1016/j.jtcvs.2009.06.023
}

exophytic obstructing tumors. ${ }^{4-6}$ APC and brachytherapy have the disadvantage that either a secondary bronchoscopy is mandatory or they do not allow the immediate opening of obstructed airways. For more detailed information regarding the advantages and disadvantages of each method, we refer the reader to the existing literature. ${ }^{7}$ The flexible cryoprobe, introduced as a tool for immediate management of airway stenosis, extends the options of interventional bronchoscopy. ${ }^{8}$ The flexible probe can be used in combination with the rigid bronchoscope to guarantee more safety during intervention and allows the use of additional techniques, such as stent implantation. Tracheal tubes allow the use of the cryoprobe in a flexible technique as well. Its $7.5-\mathrm{mm}$ working channel enables therapeutic interventions with the cryoprobe, which is $2.4 \mathrm{~mm}$ in diameter, or the combination with APC in case of bleeding. ${ }^{9,10}$

The aim of this analysis was to evaluate the efficacy and safety of the flexible cryoprobe for immediate tumor ablation in patients with exophytic endobronchial or tracheal tumor obstruction in a routine setting in a center with much expertise with the use of the flexible cryoprobe.

\section{MATERIALS AND METHODS \\ Patients}

We analyzed the data of all patients who underwent bronchoscopy in our clinic from February 2001 to September 2007. In this series we identified all patients undergoing bronchoscopic intervention with the flexible cryoprobe because of an exophytic tumor of the trachea or bronchi with subsequent 


$$
\begin{aligned}
& \text { Abbreviations and Acronyms } \\
& \begin{aligned}
\mathrm{APC} & =\text { argon plasma coagulation } \\
\mathrm{CR} & =\text { cryorecanalization } \\
\mathrm{Nd}-\mathrm{YAG}= & \text { neodymium-doped yttrium aluminum } \\
& \text { garnet }
\end{aligned}
\end{aligned}
$$

symptomatic airway stenosis. In addition to the flexible cryoprobe, a laser or APC, as well as mechanical debulking with a rigid scope, were available but rarely used since the introduction of the flexible cryoprobe in our institution in 2001. The local ethics committee approved this study.

For any bronchoscopic intervention, a platelet count of at least $100 \mathrm{Giga} /$ $\mathrm{L}$ and normal coagulation parameters (partial thromboplastin time $<40 \mathrm{sec}-$ onds and prothrombin ratio $>70 \%$ ) were mandatory. Medications influencing clotting time were stopped before intervention; heparin was stopped at least 12 hours before intervention.

\section{Cryorecanalization}

The flexible cryoprobe (Erbokryo; ERBE, Tübingen, Germany) was used as described previously. ${ }^{11}$ It can be used with the rigid bronchoscope after achievement of general anesthesia but also with the flexible technique in combination with a 7.5- or 8.5-mm Bronchoflex tube (Rüsch, Kernen, Germany), which has separate oxygen tubing, after achievement of local anesthesia and sedation. The decision of which method to apply, as well as the use of additional techniques, was left to the discretion of the treating physician and based on published guidelines. ${ }^{4,5}$ It should be noted that there is no ideal interventional strategy that can fully guarantee success and a safe outcome. The execution of any technique depends on the expertise of the team and the personal skills of the physician. If possible, we preassigned (in an expert discussion) the used interventional technique before each bronchoscopy. In cases with stenosis caused by an endoluminal tumor, almost all interventions were done as cryorecanalization (CR). In cases with mixed stenosis (with an additionally extraluminal tumor), we combined the techniques, such as CR and implantation of stents. Briefly, in general we used the following interventional technique. First, the cryoprobe is introduced through a flexible bronchoscope in combination with a flexible tube or a rigid bronchoscope. Second, the tip of the cryoprobe is pushed into the exophytic tumor mass, and freezing is started for 3 to 5 seconds with a footpad. Freezing is achieved with the Joules-Thomson effect: nitrous oxide is decompressed at the tip of the probe, which generates immediate cooling of the tip to $-89^{\circ} \mathrm{C}$. Third, the cryoprobe and the flexible bronchoscope are abruptly mechanically removed, and thus a tumor mass of up to $10 \mathrm{~mm}$ is frozen at the tip of the cryoprobe and can easily be extracted. Fourth, rapid thawing of the extracted tissue is induced in a water bath for 5 seconds. Finally, the procedure is repeated until the exophytic tumor mass has been extracted and the target bronchus is reopened. After CR, subsequent stent implantation can be performed in the same session or APC can be used to stop bleeding, if needed. Additionally, some factors could influence the intervention. Among them, bleeding of the tumor limits visibility and can significantly extend the procedural duration, which normally has a range of 15 to 60 minutes. Chest radiographic analysis was not performed routinely after the intervention and remained at the discretion of the physician.

\section{Procedural Success and Safety}

We defined our criteria to determine the success rate of CR. The bronchoscopist classified the intervention based on endoscopic criteria as (1) successful if the endobronchial tumor mass could be ablated so that either drainage of secretion or reduced airway stenosis led to a significant improvement in the patient's condition with amelioration of symptoms (eg, dyspnea, fever, or cough) and (2) not successful or a failure if the primary goal of the intervention (ie, target bronchus reopening) could not be reached.
Safety data were evaluated regarding severity of bleeding, need for a rescue operation, or prolonged mechanical ventilation for longer than 2 hours after the intervention. Bleeding was graded as mild if application of cold $\mathrm{NaCl} 0.9 \%$ solution $\left(2{ }^{\circ} \mathrm{C}-4{ }^{\circ} \mathrm{C}\right)$ or epinephrine solution $(1 \mathrm{mg} / 10 \mathrm{~mL}$ saline water) applied topically was sufficient to stop bleeding, moderate if additional techniques (APC or a bronchus blocker) had to be used, and severe if additional treatment was needed (transfusion of red blood cells, fresh frozen plasma, or coagulation factors; use of vasopressors, rescue operation; or prolonged mechanical ventilation) to stabilize the patient's condition.

\section{RESULTS \\ Patients}

Between February 2001 and September 2007, 5308 consecutive bronchoscopies were performed in our clinic. In this cohort bronchoscopic intervention with $\mathrm{CR}$ with the flexible cryoprobe for treatment of symptomatic exophytic tumor stenosis of the trachea or bronchi was used in 225 patients. The mean age of the patients was $63.9 \pm 12.9$ years (range, $19-83$ years), and 193 (85.8\%) were inpatients. One hundred fifty-six $(69.3 \%)$ patients were men.

\section{Localization and Distribution of Obstructing Tumor Lesions}

CR of obstructing tumor lesions in the central airways (trachea, main bronchus, and bronchus intermedius) was performed in $99(44.0 \%)$ patients, of the lobe bronchi in $93(41.3 \%)$ patients, and simultaneously in the central airways and lobe bronchi in $33(14.7 \%)$ patients. Multifocal endobronchial lesions (in $>1$ bronchus) were present in 67 $(29.8 \%)$ patients and were treated with the flexible cryoprobe within 1 interventional session. One hundred twenty-four $(55.1 \%)$ lesions were located in the right bronchial system, $64(28.4 \%)$ lesions were located in the left bronchial system, and $33(14.7 \%)$ lesions were located in the trachea or on the main carina. A detailed overview of lesion localizations is shown in Table 1. In 197 (87.6\%) of 225 patients, airway stenosis was malignant. One hundred forty-seven $(74.6 \%)$ patients had lung cancer. Table 2 shows the histologic findings in detail.

\section{Techniques}

The majority of interventions (194 [86.2\%] patients) were performed solely with the flexible technique. The rigid scope, always used in combination with the flexible bronchoscope, was used in $31(13.8 \%)$ patients. Additional techniques were rarely needed: APC (in patients with prolonged bleeding after CR) in $37(16.4 \%)$ patients and stent implantation in $11(4.9 \%)$ patients.

\section{Success Rate and Safety of CR}

CR was successful in $205(91.1 \%)$ of 225 interventions. In $20(8.9 \%)$ patients the intervention was not successful and did not reopen the obstructed bronchus. The main reason for unsuccessful interventions was a longer length $(>2 \mathrm{~cm})$ of the complete bronchial obstruction. 
TABLE 1. Endobronchial localization and distribution of exophytic tumor stenosis treated with cryorecanalization

\begin{tabular}{lcc}
\hline $\begin{array}{c}\text { Localization and distribution } \\
\text { of exophytic } \\
\text { tumor stenosis }\end{array}$ & $\begin{array}{c}\text { No. of } \\
\text { lesions }\end{array}$ & $\begin{array}{c}\text { Percentage of all lesions } \\
\text { treated with } \\
\text { cryorecanalization }\end{array}$ \\
\hline Right bronchial system & 131 & 58.2 \\
Left bronchial system & 68 & 30.2 \\
Trachea & 35 & 15.6 \\
Bilateral localization & 5 & 2.2 \\
Central airways & 132 & 58.7 \\
Peripheral airways & 124 & 55.1 \\
Extended lesions & 31 & 13.8 \\
Multiple localization & 49 & 21.8 \\
\hline
\end{tabular}

Note: Summery of cases might exceed the total number of cases because of overlapping lesional attribution. Bilateral localization, If cryorecanalization was performed in both the right and left bronchial system of the patient in 1 session; Central airways, trachea, left/right main bronchus, and bronchus intermedius; Peripheral airways, all other bronchi, distal of defined central airways; Extended lesions, if cryorecanalization was performed in both the central and peripheral airways in 1 session; multiple localization, if tumor stenosis was found and treated in different lobular bronchi in 1 session.

Bleeding complications occurred in $27(12.0 \%)$ patients, with mild bleeding in $9(4.0 \%)$ patients and moderate bleeding in $18(8.0 \%)$ patients. Severe bleeding did not occur. Thus the overall bleeding rate was low. No case of pneumothorax was detected. One manifestation of pneumomediastinum was seen in a patient with a cystadenoma, a benign tracheal tumor. In this patient injury of the pars membranacea occurred after removal of the bulky tumor. The patient was hospitalized and treated with antibiotics to prevent mediastinitis. Surgical intervention was evaluated but not necessary after spontaneous closure of the very small tracheal perforation. The patient fully recovered after 5 days without further complications.

\section{DISCUSSION}

Patients with symptomatic bronchial obstruction caused by an endoluminal exophytic tumor require immediate treatment. Selection of the appropriate interventional technique depends on the treatment plan (curative or palliative intention) and on the type of tumor stenosis (endoluminal, extraluminal, or mixed). ${ }^{5}$ The combination of both rigid and flexible bronchoscopy is the most commonly and widespread used method, and it allows additional use of APC, an Nd-YAG laser, electrocautery, or CR. The main advantage of the laser, electrocautery, and CR is their immediate effect by tumor ablation. The flexible cryoprobe allows recanalization within a single intervention without the necessity of a secondary bronchoscopy. The cryoprobe can be resterilized and reused without any restrictions in effectiveness. CR or tumor debulking with a flexible cryoprobe performed in the way that is described in this article is a relatively new approach. The previously performed feasibility study in our institution of 60 patients showed a high success
TABLE 2. Overview on histologic findings

\begin{tabular}{lc}
\hline \multicolumn{1}{c}{ Conditions } & Patients, no. (\%) \\
\hline Nonmalignant lesions* & $28(12.4)$ \\
Malignant lesions & $197(87.6)$ \\
$\quad$ Lung cancer & $147(74.6)$ \\
$\quad$ SCLC & $26(17.7)$ \\
$\quad$ NSCLC & $121(82.3)$ \\
$\quad$ Adenocarcinoma & $13(10.7)$ \\
$\quad$ Squamous cell cancer & $83(68.6)$ \\
$\quad$ Other or not specified NSCLC & $25(20.7)$ \\
Other malignant lesions $\dagger$ & $50(25.4)$ \\
\hline SCLC, Small cell lung cancer; NSCLC, non-small cell lung cancer. *Nonmalignant \\
lesions were as follows: benign tracheal stenosis, lipoma, endobronchial granulation \\
tissue, and hamartoma. $\dagger$ Other malignant lesions were (number and percentage \\
of all malignant lesions) as follows: colorectal cancer (17 [8.63\%]), carcinoid \\
(6 [3.05\%]), lymphoma (6 [3.05\%]), breast cancer $(5[2.54 \%])$ ovarian carcinoma \\
(4 [2.03\%]), esophageal carcinoma (4 [2.03\%]), renal cell carcinoma (3 [1.52\%]), \\
malignant melanoma (2 [1.02\%]), thyroid cancer (2 [1.02\%]), and laryngopharyngeal \\
carcinoma (1 [0.51\%]).
\end{tabular}

rate $(83 \%)$ of CR with a moderate bleeding rate of $10 \%{ }^{8}$ The present study confirms the findings of the feasibility study and showed that even under daily routine conditions in the bronchoscopy suite, CR of exophytic endobronchial tumor stenoses is safe and successful in about $91 \%$ of cases. The success rate in our present study was even higher than in the feasibility study. ${ }^{8}$ This might be explained by the learning curve of the $\mathrm{CR}$ procedure. A current chest computed tomographic scan to estimate the distance and complexity of the stenosis is mandatory before intervention to ensure safety and for the appropriate selection of patients. We tried to reopen longer tumor stenoses than were seen on the computed tomographic scan. However, in these cases a lower success rate has to be taken into account. This was also the case in our study, in which the main reason for unsuccessful intervention was a long tumor stenosis with infiltrative tumor growth. The duration of pre-existing tumor stenosis (as measured from the onset of symptoms) did not seem to influence the procedural success rate, even if partial atelectasis or mediastinal shift was seen. It was frequently possible to reopen 4- to 6-week-old tumor obstructions successfully by using $\mathrm{CR}$. $^{11}$

We found a higher rate of moderate compared with mild bleeding in our study. Because the freezing has a hemostatic effect on minor bleeding lesions, it is likely that this is not sufficient in cases with severe tumor-associated bleeding. On the other hand, mild bleeding often occurs during bronchoscopic interventions in patients with lung cancer and is not always routinely reported as an adverse event. Therefore it can be assumed that the rate of mild bleeding is underestimated with conventional bronchoscopic techniques. However, with strict attention to normal coagulation parameters and platelet counts before the intervention, there was no severe bleeding complication in our study. For safety reasons, CR was not done if chest computed tomographic scans showed tumor involvement of large vessels in the interventional region. 
An important advantage of this new approach of CR with the flexible cryoprobe is that it can easily be performed with both flexible and rigid techniques. Compared with the Nd-YAG laser, which is still the standard technique in large institutions, cost for purchase, repair, and maintenance for the less invasive techniques of APC, electrocautery, and $\mathrm{CR}$ are lower. ${ }^{5}$ A cost-effectiveness study revealed that the average treatment cost for the Nd-YAG laser was $20 \%$ higher compared with that for electrocautery, which was caused by a longer hospital stay before bronchoscopic treatment. ${ }^{12}$ Another advantage of CR is that large particles of tumor can be extracted with the flexible cryoprobe. Thus the time for effective tumor mass ablation is generally shorter compared with that for laser therapy. The shorter intervention time is one of the reasons why so many CRs in our clinic could be performed with the flexible bronchoscopy technique. Furthermore, the study shows clearly that the use of a tracheal tube as a working channel (instead of the rigid scope) is a feasible and safe option and allows a completely flexible interventional technique.

APC, which is also used for treatment of malignant airway stenosis, requires a second bronchoscopic intervention for the removal of devitalized tissue 48 to 72 hours after initial APC treatment. ${ }^{10}$ We achieved a higher procedural success rate compared with that seen after APC. Reichle and colleagues ${ }^{13}$ showed the efficacy of APC in 186 patients with malignant airway stenosis. They achieved "good results" in only $67 \%$ of cases. Moreover, one has to take into account that in this study more than $90 \%$ of interventions were performed with rigid bronchoscopy. In contrast, the rigid bronchoscope was used in only $13.8 \%$ of interventions in our study. However, APC and CR can be effectively combined and do not exclude each other. We successfully used APC to stop bleeding after application of the cryoprobe in $16.4 \%$ of patients.

In conclusion, CR is a safe and successful method for endobronchial exophytic tumor debulking. The flexible cryoprobe is easy to use and allows rapid removal of exophytic tumors under direct observation with low bleeding rates. CR can be regarded as an alternative primary tool and complementary method to APC, electrocautery, or the Nd-YAG laser for endobronchial tumors. This study showed promising safety data for the routine use of the flexible cryo- probe over the long-term. The main advantage of CR is its immediate effect on tumor stenosis with subsequent rapid improvement of the patient's symptoms. For future studies, we recommend additionally evaluating patient-related outcomes with the dyspnea scale and quality-of-life instruments.

The purpose of this nonrandomized study was to describe the safety and efficacy of a flexible cryoprobe as a primary tool for endobronchial recanalization in malignant lesions. As a retrospective study, a per-protocol patient selection was not conducted. However, a prospective randomized study will follow to compare different interventional methods regarding safety, efficacy, and selection strategies for each technique.

\section{References}

1. Salajka F. Occurrence of haemoptysis in patients with newly diagnosed lung malignancy. Schweiz Med Wochenschr. 1999;129:1487-91.

2. Vaaler AK, Forrester JM, Lesar M, Edison M, Venzon D, Johnson BE. Obstructive atelectasis in patients with small cell lung cancer. Incidence and response to treatment. Chest. 1997;111:115-20.

3. Soyseth V, Benth JS, Stavem K. The association between hospitalisation for pneumonia and the diagnosis of lung cancer. Lung Cancer. 2007;57:152-8.

4. Bolliger CT, Mathur PN, Beamis JF, Becker HD, Cavaliere S, Colt H, et al. ERS/ ATS statement on interventional pulmonology. European Respiratory Society/ American Thoracic Society. Eur Respir J. 2002;19:356-73.

5. Bolliger CT, Sutedja TG, Strausz J, Freitag L. Therapeutic bronchoscopy with immediate effect: laser, electrocautery, argon plasma coagulation and stents. Eur Respir J. 2006;27:1258-71.

6. Vergnon JM, Huber RM, Moghissi K. Place of cryotherapy, brachytherapy and photodynamic therapy in therapeutic bronchoscopy of lung cancers. Eur Respir J. 2006;28:200-18.

7. Ernst A, Feller-Kopman D, Becker HD, Mehta AC. Central airway obstruction. Am J Respir Crit Care Med. 2004;169:1278-97.

8. Hetzel M, Hetzel J, Schumann C, Marx N, Babiak A. Cryorecanalization: a new approach for the immediate management of acute airway obstruction. $J$ Thorac Cardiovasc Surg. 2004;127:1427-31.

9. Schumann C, Kropf C, Wibmer T, Merk T, Kruger S. Therapy of exophytic bronchial tumorous stenosis by flexible cryoprobe. Eur Respir J. 2006;28:1286-7.

10. Morice RC, Ece T, Ece F, Keus L. Endobronchial argon plasma coagulation for treatment of hemoptysis and neoplastic airway obstruction. Chest. 2001;119: 781-7.

11. Schumann C, Lepper PM, Barth TF, Moller P, Kruger S. Successful immediate cryorecanalization of a simultaneous high-grade tracheal and bronchial stenosis as rare manifestations of bronchial-associated lymphoid tissue lymphoma. $J$ Thorac Cardiovasc Surg. 2009;137:e17-9.

12. Boxem T, Muller M, Venmans B, Postmus P, Sutedja T. Nd-YAG laser vs bronchoscopic electrocautery for palliation of symptomatic airway obstruction: a costeffectiveness study. Chest. 1999;116:1108-12.

13. Reichle G, Freitag L, Kullmann HJ, Prenzel R, Macha HN, Farin G. [Argon plasma coagulation in bronchology: a new method-alternative or complementary?]. Pneumologie. 2000;54:508-16. 